\title{
Verflochtene Sprachen und Kulturen: Gohar Markosjan-Käspers Romane als Beispiele des mehrsprachigen Schreibens
}

\author{
AIGI HEERO
}

\begin{abstract}
Intertwined Languages and Cultures: Gohar Markosjan-Käsper's Novels as Examples of Multilingual Writing. The present article analyses the phenomenon of multilingualism in the novels of Gohar Markosjan-Käsper (1949-2015) and discusses her life and work in the socio-political context of the former Soviet Union (in relation to language and cultural politics). Markosjan-Käsper was an Armenian-born writer who spent most of her life in Estonia and wrote her books in Russian. Accordingly, her works originated in a contact zone of different languages and cultures. This article highlights her novels Helena and Penelopa as examples of transcultural writing and analyses the manifestations and functions of multilingualism in these works. The study shows that a number of topics and motifs that are present in German-language transcultural literature also appear in Markosjan-Käsper's novels (for example cultural comparison, self-discovery in a foreign culture). The multilingualism can be seen in these novels both explicitly and implicitly: in addition to Russian, other languages such as Armenian, Estonian, English, and Latin are used, with numerous indirect references to these languages. Furthermore, various references to world-famous novels such as Ulysses by James Joyce and Master and Margarita by Michail Bulgakov are analysed.
\end{abstract}

Keywords: Gohar Markosjan-Käsper; multilingualism; transcultural literature; Estonian literature; Armenian literature; Soviet Union; language politics; cultural politics

\section{Einleitung}

Im März 2002 erschien in Berlin der Roman Penelopa von Gohar MarkosjanKäsper (1949-2015) in deutscher Übersetzung (mit dem Titel Penelope, die Listenreiche). Die Autorin stammte aus einer armenischen Künstlerfamilie in Jerewan: Ihre Mutter war Balletttänzerin, ihr Vater Opernsänger. Sie studierte Medizin und arbeitete etliche Jahre als Ärztin, doch schon seit ihrer Jugend schrieb sie Gedichte und versuchte sich auch an Prosatexten. 1990 heiratete sie 
den estnischen Schriftsteller und Übersetzer Kalle Käsper, den sie in Armenien kennengelernt hatte und übersiedelte nach Estland. Danach widmete sie sich ganz der Literatur.

Obwohl sie armenischer Herkunft war, verfasste Markosjan-Käsper ihre Texte auf Russisch. Hierbei hat wohl die Tatsache, dass sie die russische Sprache als Sprache des Selbstausdrucks betrachtete, die wichtigste Rolle gespielt. Als Kind hat sie in Jerewan die russischsprachige Schule besucht und dadurch gründliche Kenntnisse dieser Sprache erworben. Des Weiteren wurde auch in ihrer Familie Weltliteratur in russischer Übersetzung gelesen und teilweise auf Russisch kommuniziert. Auf diesen Aspekt wird später in dieser Studie etwas näher eingegangen.

An dieser Stelle muss jedoch präzisiert werden, dass ihre Familie sowie ihre Sprachenwahl in dieser Hinsicht eine Ausnahme darstellen. MarkosjanKäsper hat selber erklärt, dass am Ende der 1970er Jahre in Armenien etwas wie die russischsprachige Literatur initiiert wurde. Es habe aber ziemlich wenige solche Autoren gegeben, und deren Schaffen habe nicht besonders viel Aufmerksamkeit bekommen (Markosjan-Käsper 2006: 69). Der Grund dafür, so Markosjan-Käsper, lag wohl daran, dass die armenische literarische Tradition eine sehr lange Geschichte hat und die neue, russischsprachige Literatur einerseits als nicht qualitativ hochwertig betrachtet wurde und andererseits sah man daran auch ein Anzeichen der Sowjetisierung (ebd.). Obwohl die Sowjetmacht versuchte, Russisch in Armenien als offizielle Sprache einzuführen, wurde dieses Ziel nicht erreicht. Dennoch wuchs die Rolle des Russischen im öffentlichen Leben:

A thorough knowledge of Russian was a virtual requirement for white collar jobs, and some parents preferred to send their children to Russian-language schools. A portion of Armenian citizens opted for higher education in Russian universities and technical schools. (Grenoble 2003: 123)

Dessen ungeachtet blieb der Anzahl der armenischen Bürger, die Russisch als ihre erste Sprache betrachteten, relativ gering. Russisch als Zweitsprache dagegen war viel weiter verbreitet: 1989 gaben 60,6\% der Armenier Russisch als ihre zweite Sprache an (ebd.: 135). Die starke literarische Tradition, die muttersprachliche Hochschulbildung und das starke Nationalbewusstsein der Armenier trugen dazu bei, dass der Einfluss des Russischen auf armenische Kultur geringfügig blieb und dass die russische Sprache für Armenier nicht ein Teil ihrer Identität ist (vgl. Rabanus/Barseghyan 2015: 24-25).

Markosjan-Käspers literarische Texte entstanden meist in Estland, wurden jedoch meistens in Russland erstmals publiziert. Der Grund dafür ist vermutlich rein pragmatisch gewesen: Die Möglichkeiten, in Estland ein russisch- 
sprachiges Buch herauszugeben, waren in den 1990er und den 2000er Jahren eher spärlich. Estnische Autoren, die auf Russisch schrieben, orientierten sich deshalb auf Russland und seine Literatur, um eine wesentlich größere Leserschaft zu gewinnen (vgl. Kotjuh 2012: 139).

Penelopa war Markosjan-Käspers erster Roman, der 1998 in St. Petersburg erschien. Es folgten weitere Romane wie Helena (2000, auf Estnisch 2003; eine deutsche Übersetzung liegt nicht vor), Karyatiden (2003), die Novellensammlung Der Traum (2006) und viele andere Werke. 2012 erschien ihr letzter, autobiographischer Roman Memento mori. Sie starb 2015 nach einer schweren Krankheit.

Die Leserschaft sowie die meisten Kritiker reagierten auf MarkosjanKäspers Werke positiv, sogar lobend: Man charakterisierte ihre Schreibweise als mutig und meisterhaft, reich an Wortspielen und Assoziationen, als einen Ausdruck des „magischen Realismus“ (Zirnask/Püve 2005). Tatsächlich vermischen sich in ihren Werken verschiedene Realitäten, Schauplätze und kulturelle Schichtungen. Was diese zwei Werke dabei ganz besonders auszeichnet, ist die geschickte Verwendung und Vermischung von verschiedenen Sprachen: Die Originalsprache der Romane, Russisch, geht je nach Bedarf fließend ins Armenische, ins Lateinische, ins Englische oder ins Estnische über. Aus diesen Gründen (Verflechtung von Schauplätzen und Kulturen sowie die Präsenz der Mehrsprachigkeit) wurde Markosjan-Käsper selbst mal als armenische, mal als russische oder russischsprachige, oder mal als estnische russischsprachige Schriftstellerin bezeichnet (vgl. Kartau 2015).

Eine umfassendere Abhandlung über Markosjan-Käspers Werke gibt es bis jetzt leider nicht (abgesehen von kleineren Artikeln in der Tagespresse oder kurzen Erwähnungen in Überblicksartikeln). Die vorliegende Studie versucht deshalb, dieses Desiderat zu beheben und ihre Werke in den internationalen Kontext zu stellen. Als erstes werden ihre Romane als Beispiele des transkulturellen Schreibens analysiert. Dafür sprechen etliche Gründe. MarkosjanKäsper verfasste ihre Werke auf Russisch, jedoch verstand sie sich nicht als russische Schriftstellerin, sondern positionierte sich als Autorin, deren Schaffen durch vielfältige kulturelle Einflüsse beeinflusst ist:

Und generell liegt meiner Seele die französische Literatur am nächsten, ich halte sie für die größte Literatur der Welt [...]. Ich lese immer wieder die Werke von Balzac, Zola, Proust und von vielen anderen französischen Schriftstellern. Sicher, ich lese immer wieder auch Dostojevskij und Bulgakov. Und dennoch kann ich nicht sagen, dass alle diese Autoren mich inspiriert haben, denn die Inspiration kann man nur von der Perfektion bekommen, [...] (MarkosjanKäsper 2006: 66) 
HEERO

Also können wir festhalten, dass Gohar Markosjan-Käsper zweisprachig aufgewachsen ist und durch ihre (mehrsprachige) Belesenheit vielfältige kulturelle Impulse erhalten hat. Gleichzeitig hat sie nie ihren armenischen kulturellen Hintergrund aufgegeben, auch wenn sie einen sehr großen Teil ihres Lebens in Estland verbracht hat. So sind in ihren Werken viele Themen und Züge erkennbar, die auch in Werken von deutschsprachigen transkulturellen Autoren vorhanden sind. Diese Züge werden im Folgenden hervorgehoben und im Vergleich mit einigen transkulturellen Autoren aus Deutschland dargestellt. Des Weiteren wird auf den Begriff der mehrsprachigen Literatur eingegangen und Markosjan-Käspers Romane in diesem Kontext analysiert. Im Fokus dieser Studie stehen die Romane Penelope, die Listenreiche (2002) und Helena $(2003)^{1}$.

\section{Gohar Markosjan-Käsper als Autorin transkultureller Literatur}

Wenn wir über Literatur, die von Menschen mit Migrationshintergrund geschaffen wurde, sprechen, denken wir an die früheren Begriffe wie „Gastarbeiterliteratur“, „littérature des immigrations“, „littérature des Beurs“, „Black British Literature“ oder an jüngere Termini wie „Hybridität“, „transkulturelle Literatur“ oder gar an „neue Weltliteratur“ (Glesener 2016). Implizit wird dabei wohl an Literatur in den großen Weltsprachen gedacht. Viel weniger hat die globale Forschung ihre Aufmerksamkeit den kleineren Sprachen und Literaturen gewidmet. Es mag vielleicht überraschend sein, doch hat auch die Literatur in Estland eine sehr lange Tradition des multikulturellen Schreibens, denn die estnischsprachige literarische Kultur nahm ihren Anfang im Kontaktfeld von mehreren Sprachen und Kulturen (Heero 2019: 144-146).

Neben der deutschsprachigen (Lukas 2008: 23-32) hat auch die russischsprachige Literatur in Estland (estn. vene kirjandus Eestis) eine lange Tradition. In der estnischen Literaturgeschichtsschreibung wird ihr Beginn auf das Jahr 1918 gesetzt (die Gründung der Estnischen Republik). Die russischsprachige Literatur, die davor geschaffen wurde, zählt man im Allgemeinen zu der Literatur des Russischen Imperiums; bei diesen literarischen Texten ist kaum ein spezifischer Estland-Bezug festzustellen (Belobrovtseva 2018: 102). Es sollte vermerkt werden, dass die Geschichte dieser Literatur eng

1 Die Zitate aus dem Roman Helena werden im Text mit dem Kürzel H und der Seitenangabe, die Zitate aus dem Roman Penelope, die Listenreiche mit dem Kürzel P und der Seitenangabe, das Zitat aus der russischen Ausgabe von „Пенемопа“ wird mit dem Kürzel PII und der Seitenangabe versehen. Alle Zitate aus Helena sind von mir ins Deutsche übertragen worden. 
mit der Geschichte der Migration aus Russland (später aus den Gebieten der Sowjetunion) verbunden (ebd.: $110-118)^{2}$ und das Schaffen der russischen Literaten in Estland oft den russischen Literaturtraditionen verpflichtet ist. Erst zu Beginn des 21. Jahrhunderts hat man angefangen, diese Literatur als ein Beispiel des Schreibens zwischen den Sprachen und Kulturen zu kontextualisieren (ebd.: 119). Markosjan-Käspers Werk Helena, in dem die Migrationserfahrung einer Frau in Estland der 1980er und 1990er Jahren beschrieben wird, lässt sich daher als Beispiel des transkulturellen Schrifttums darstellen. Es soll dabei aber betont werden, dass die Geschehnisse dieses Romans aus der Perspektive eines auktorialen Erzählers dargestellt werden. Die Heldin erscheint wie ein Untersuchungsobjekt, an dem ein Versuch durchgeführt wird, um die Frage, ob sie in einer neuen Kultur zurechtkommt oder nicht, zu beantworten. Dieser Versuch wird von dem Erzähler genau beobachtet, (vergleichend) analysiert und teilweise humorvoll kommentiert.

Als transkulturelle Literatur wird im Kontext dieser Studie im Allgemeinen das Schaffen der Autoren verstanden, die ihre Werke in einer Sprache verfassen, die nicht ihre Muttersprache ist und die die Erfahrung des kulturellen AndersSeins reflektieren. In der Literaturwissenschaft wurden in den 1960 und den 1970er Jahren solche Werke als „multi- oder interkulturelle“ Literatur bezeichnet, mit der „literarisch ausgestalteten Identitätsproblematik“ als Hauptthema

2 In Bezug auf die Migration aus Russland (ab 1922 aus der Sowjetunion) nach Estland in den Jahren 1918-1939 ist festzustellen, dass Estland in dieser Zeit hauptsächlich eine Rolle des Transitlandes spielte: Man flüchtete vor bolschewistischem und später vor stalinistischem Terror nach Westeuropa und das naheliegende Estland diente als Stützpunkt (vgl. Belobrovtseva 2018: 103). Anders verhielt sich mit der Migration aus den Sowjetrepubliken nach dem II. Weltkrieg. Unmittelbar nach Kriegsende (um 1945-46) kamen hauptsächlich Industriearbeiter nach Estland, denn gleich nach dem Krieg fand dort ein Prozess der forcierten Industrialisierung statt. Die Einwanderung erreichte in den Nachkriegsjahren einen Höhepunkt im Jahr 1955, in dem etwa 45000 Migranten in Estland eintrafen. Das machte etwa 4\% der gesamten Bevölkerung des Staates in diesem Jahr aus (Sakkeus 1994). Die nächste größere Immigrationswelle nach Estland fand in den 1960er Jahren statt und gipfelte im Jahr 1970, in welchem etwa 30000 Migranten vor allem aus ferneren Gebieten im Osten und Süden der Sowjetunion ankamen (insgesamt 2,2\% der Gesamtbevölkerung) (vgl. ebd.). In den 1960-70er und 1980er Jahren spielte jedoch die freiwillige Arbeitsmigration die Hauptrolle. Hinzu kamen Studenten und Offiziere, die nach dem Dienst in Estland bleiben wollten, Arbeiter im Bereich der ideologischen Agitation und Propaganda sowie die Menschen, die durch Heirat nach Estland kamen (Lember 2017). Um 1989 betrug der Anteil der Einwanderer der ersten Generation 26\% der Bevölkerung, dazu kamen $10 \%$ der Vertreter der zweiten, also in Estland geborenen Generation, insgesamt 36\% (Katus/Puur/Põldma 2002). 
(Baumgärtel 1997: 54). Die Autoren wurden auf der Grundlage eines statischen Identitätskonzepts gerne als Personen dargestellt, die ständig auf der Suche nach ihrer Identität sind und unter dem Leben in der Fremde leiden (Amodeo 1996: 42). Diese Thematik ist heute wohl nicht mehr ganz aktuell. Deshalb haben sich auch die Begriffe wie Literatur der Transkulturalität, oder sogar der Transdifferenz durchgesetzt. Diese überwinden die binären Grenzziehungen zwischen den Kulturen und implizieren die Tatsache, dass die zeitgenössischen Kulturen denkbar stark miteinander verbunden und verflochten sind: Es geht um die wechselseitige Überlagerung von kulturellen Zugehörigkeiten innerhalb der sichtbaren Differenzen. Dabei wird die Differenz nicht aufgehoben, sondern das kulturelle Anderssein wird als etwas Positives hervorgehoben und bewusst gepflegt (Allolio-Näcke/Kalscheuer/Manzeschke 2005: 27).

In der transkulturellen Literatur werden diese Erfahrungen des Lebens zwischen zwei Kulturen oft thematisiert und es wird der Prozess der Aneignung einer neuen Kultur literarisch analysiert. Diese kulturelle Selbstfindung bzw. Selbstreflexion wird oft auf konkrete Orte bezogen, was auch mit der „Erfahrung des Unterwegsseins“ verbunden sein kann (Heero 2009: 208-209). Tallinn, die estnische Hauptstadt erscheint in Helena anfangs kalt, anonym und einer typischen skandinavischen Großstadt ähnlich. Die Menschen, die dort leben, scheinen an ihrer Gegenwart zu leiden, sie sind ständig gehindert, ihre Träume oder beruflichen Ehrgeiz auszuleben und gleichzeitig damit beschäftigt, ihren Alltag zu bewältigen (ebd.: 216-217). Auch Helena möchte in Tallinn als Heilmedizinerin praktizieren, kann es aber nicht, denn sie beherrscht nicht die Landessprache und es gelingt ihr nicht, in einer kulturell fremden Umgebung ein soziales Netzwerk aufzubauen:

Wenn man in Armenien einen Menschen kuriert, schickt er zehn neue zu dir, er spricht mit allen darüber, lobt dich mehr als eine Werbeagentur, seine Verwandten, Freunde, Kollegen werden dann geradezu deine Tür einrennen [...]. In Estland konntest du eine Leiche auferstehen lassen, und keine Seele hat davon erfahren, die Leiche jedenfalls teilte den anderen diese Nachricht nicht mit, nach der Auferstehung aus dem Grab ging sie am nächsten Tag ruhig zur Arbeit und wenn jemand fragte, wie es ihr gelang, wieder aufzustehen, lächelte sie nur geheimnisvoll. $(\mathrm{H}, 123)$

Doch mit der Zeit lernt sie die Schönheit der Tallinner Altstadt und der estnischen Landschaften zu schätzen. Sie studiert gründlich die Verhaltensmuster von Esten in verschiedenen Kommunikationssituationen und dank dessen kann sie ihr eigenes Verhalten anpassen und Freundschaften schließen. Das Ende der Sowjetunion jedoch und die damit gebundenen gesellschaftlichen Umwälzungen kann sie schwer verkraften (womit sie das Lebensgefühl von 
vielen Menschen in Estland teilt). Von seinem Gatten, Olev, bekommt sie wenig moralische Unterstützung, und es scheint, dass mit dem Anbruch der neuen Zeiten auch ihre Liebe erlöscht. Deshalb scheint es für sie logisch und natürlich, zurück zu den Wurzeln zu kehren und in Jerewan nochmals neu anzufangen.

Etliche Male werden auch kulturelle Vergleiche zwischen Armenien und Estland gezogen. So charakterisiert Helena von ihrem kulturellen Standpunkt aus gesehen die estnischen Frauen als „blass, ausdruckslos, gar hässlich“ und wundert sich, wie diese so einfach einen Mann aus dem Westen finden $(\mathrm{H}, 81)$. An einer anderen Stelle beschreibt sie die Esten als „eisig“ und „formell“, sie „gingen im gleichmäßigen Rhythmus, wedelten nicht mit Händen, doch ihre Langsamkeit kompensierten sie mit einem wilden Sprechtempo." (H, 72-73) Gleichzeitig bewundert sie die Selbstbewusstheit der Estinnen und die souveräne Art, mit der sie mit Beziehungen umgehen. Ganz bewusst versucht sie, mit ihren „armenischen Vorurteilen“ $(H, 82)$ zu kämpfen. Deshalb möchte Helena nicht nur als Zuschauerin dastehen, sondern sich am estnischen Leben aktiv beteiligen und auch die estnische Sprache lernen. Diese Sprache entpuppt sich aber als schwierig. Des Weiteren entdeckt sie, dass es ihr zwar leicht fällt, etwa unter Kollegen neue Bekanntschaften zu finden, aber es ist für sie als eine eher geschlossene Person schwierig, wirklich tiefe und bedeutende Freundschaften zu schließen $(H, 117)$. Deshalb bleibt sie bis zum Ende ihres Aufenthalts in Tallinn eher Außenseiterin, die das dortige Leben mit innerer Distanz betrachtet. Gleichzeitig erkennt und akzeptiert sie sowohl ihr kulturelles Anderssein und als auch ihre kulturell hybride Identität: Ihre Muttersprache ist armenisch, doch hat sie (wie auch Markosjan-Käsper selber) eine russische Schule besucht und empfindet deshalb Russisch als die Sprache des intellektuellen Austausches und des Selbstausdrucks: „Wenn es notwendig war, Gedanken und Überlegungen zu verbalisieren, ging sie unbewusst zur russischen Sprache über" (H, 106). Sie ist dessen bewusst, dass die Esten diese Sprache am Ende der sowjetischen Okkupation als „die Sprache des Feinds“ empfinden, aber für sie ist die Benutzung des Russischen wie ein „intellektueller Orgasmus“ ( $\mathrm{H}$, 107). Vor dem Hintergrund einer anderen Kultur versteht sie auch, dass sie Kosmopolitin ist, die sich für Opernmusik, klassische Literatur, russisches Theater und die Philosophie Asiens interessiert. Also kann man behaupten, dass die Heldin nach einem anfänglichen Kulturschock wieder einen Weg zu sich selbst findet und ihr Wesen als Ausdruck eines transdifferenten Daseins akzeptiert. 


\section{Zur Funktion der Mehrsprachigkeit in Gohar Markosjan- Käspers Romanen Helena und Penelopa}

Die hybriden/globalen Identitäten können sich in Phänomenen wie (literarischer) Mehrsprachigkeit und Mehrfach-Identitäten manifestieren. Die Mehr- und Vielsprachigkeit sollten in diesem Fall als nichts Exotisches, sondern als etwas Natürliches betrachtet werden (Ernst 2019: 92-93). Je nach Bedarf können in einem Text verschiedene Sprachen nebeneinanderstehen. In der jüngeren Forschung benutzt man für solche Texte auch den Begriff „exophone Literatur", das ist die Literatur, die in einer Zweitsprache geschrieben worden ist, doch dennoch Spuren einer ersten Sprache aufweist und diese thematisiert. Dies kann auf rein oberflächlich linguistischer Ebene stattfinden, doch kann auch durch Mittel wie Code-Switching oder indirekte Übersetzung in der Zweitsprache ganz neue, kreative Räume öffnen und stilistische Register schaffen (Wright 2008: 39-40).

Die Wurzeln einer solchen Betrachtungsweise liegen jedoch wohl bei dem sogenannten postmodernen Turn in den 1980er Jahren, der die traditionelle Zeit- und Raumauffassung relativierte. Statt über eindimensionale Assimilationsmodelle sprach man über multidimensionale (darunter auch multilinguale) Bewegungen zwischen Geburtsland und Aufenthaltsland (Jürgenson 2016: 95; Darieva 2007: 78-79). Das heißt, die Identität ist nicht geographisch-kulturell determiniert, sondern oft gebunden an soziale Interaktion und an die Bewegung zwischen unterschiedlichen Diskursen (Martinez Guillem 2015: 3-4). So kann die Literatur zu einer Manifestation von verschiedenen Sprachen und Kulturen werden. Dembeck und Parr etwa sehen den Vorteil einer solchen Betrachtungsweise darin, dass man dadurch den (literarischen) Text selbst in den Fokus nimmt und ihn nicht mehr von textexternen Faktoren wie der Migrationserfahrung aus betrachtet (Dembeck/ Parr 2017: 9-10). Diese Betrachtungsweise ermöglicht auch, bestimmte Vorurteile oder Erwartungshaltungen, die das Deuten eines Textes (leider) des Öfteren beeinflussen, beiseite zu lassen. ${ }^{3}$

Laut Dembeck und Parr können wir über die explizite Mehrsprachigkeit reden, wenn in einem Text Segmente aus diversen Sprachen nebeneinanderstehen, so dass die unterschiedlichen Idiome klar voneinander unterscheidbar sind (Dembeck/Parr 2017: 10). Auch Markosjan-Käsper verwendet in ihren Romanen neben dem Russischen bruchstückartig auch andere Sprachen (etwa

\footnotetext{
Zum Beispiel wurde Markosjan-Käspers Penelope, die Listenreiche in einer Rezension der Neuen Zürcher Zeitung vorgeworfen, dass man dort nur wenig über die leidvolle Geschichte der Armenier des 20. Jahrhundert erfahren habe (Spengler-Axiopoulos 2002: 67).
} 
das Armenische), um spezifische Erscheinungen, wie z.B. bestimmte Speisen, zu markieren: „[...] was jeder so draufhat, der eine die ewigen adschapsan$\mathrm{dal}^{4}$ und boschbasch ${ }^{5 \text { “ }}(\mathrm{P}, 175)$. Auch bei Begriffen, die bestimmte kulturelle Konzepte in sich tragen, keine exakte Entsprechung in anderen Sprachen haben und deshalb unübersetzbar sind, verwendet sie den Originalbegriff aus der anderen Sprache: „Der Mann soll sich vom ersten Tag an als Hausherr fühlen. Und das ist bei diesen Schwiegereltern nicht möglich. Aus einem tanpessa ${ }^{6}$ kann doch nur ein Gnadenbrotempfänger werden." (P, 215). Des Weiteren finden sich in ihren Büchern Überschriften, Buchtitel oder explizite Zitate auf Armenisch, etwa „Der Schöpfer des einzigartigen Verses ,Aschnan mscheschum schrschuk u scherschjun ${ }^{67}$ und kommunistisches Geschrei, Gelärm, Gefeuer?" (P, 61-62).

Weiterhin wird in dem literarischen Werk eine andere Sprache eingesetzt, um zu explizieren, dass eine Person eine Fremdsprache spricht, um die Eigenartigkeit des fremdsprachlichen Sprechens zu unterstreichen (Dembeck/ Parr 2017: 10). Markosjan-Käsper benutzt beispielsweise in Helena estnische Wörter und Wendungen um mit diesen gebundene kulturelle Verhaltensweisen $\mathrm{zu}$ illustrieren. „Sie behandelte [...] eine ältere schwatzhafte Russin, die immer nach drei Worten das estnischsprachige ,kurat' hinzufügte, [...] und einen estnischen jungen Mann, der außer ,tere' und ,head aega' ${ }^{6}$ kein einziges Wort in keiner Sprache sagte“ (H, 131). Illustriert werden hier sowohl die Wortkargheit der Esten als auch bestimmte Sprechweise der Nicht-Esten, die die Landessprache nicht besonders gut beherrschen.

Englisch wird in diesem Roman als eine inhaltslose Sprache „des Bill Gates" (H, 96), der kapitalistischen Politik und Bürokratie empfunden und das Sprechen auf Englisch mit zu schneller Amerikanisierung assoziiert $(\mathrm{H}$, 97-98). Helena sieht mit Befremden, wie Politiker, die dem Englischen nicht besonders mächtig sind, die Sprache trotzdem sprechen, und zwar mit einem starken Akzent: „[...] haben doch die stolzen Präsidenten der drei baltischen Staaten miteinander auf Englisch kommuniziert, wenn sie auf einem sammit ${ }^{9}$

\footnotetext{
Gemüseeintopf (vgl. P, 393).

Suppe mit Fleisch (vgl. P, 393).

Eingeheirateter Mann, der von Schwiegereltern ausgehalten wird (vgl. P, 394).

7 Beginn eines Gedichts von Wahan Terjan, einem armenischen Lyriker, der auch Mitglied der kommunistischen Partei war (vgl. P, 390).

8 kurat=Teufel, estnisches, nicht besonders starkes Schimpfwort, das sehr häufig benutzt wird; eines der Wörter, das von Nicht-Esten sehr schnell übernommen wird, auch wenn sie die Sprache sonst nicht sprechen; tere = hallo; head aega = auf Wiedersehen.

9 Die Autorin benutzt hier diese Schreibweise des englischen Wortes „summit“, um den starken estnischen Akzent im Englischen zu betonen. (Hervorhebung A.H.)
} 
zusammentrafen." (H, 97). Ähnlich wirken auch literarisch-kulturelle Anspielungen auf Englisch. So wird indirekt auf das Schauspiel Pygmalion von George Bernard Shaw bzw. auf das Musical My Fair Lady von Frederick Loewe und Alan Jay Lerner verwiesen: „[...] Liana stoppte plötzlich und begann, mit der Begleiterin des Wikingers [Olev] unisono zu schwätzen, indem sie den üblichen, aus Standardausdrücken bestehenden Begrüßungswechsel durchführte (how do you do, mrs. Higgins)" (H, 30). Damit wird auf die Inhaltslosigkeit des Small-Talks und die Oberflächlichkeit der Konversation gedeutet - was einerseits mit der literarischen Anspielung konvergiert, andererseits aber auch die eher kritische Einstellung gegenüber der englischsprachigen (die in diesem Roman weitgehend mit der amerikanischen gleichgesetzt wird) Kultur, Gesellschaft und Politik verdeutlicht.

Sehr oft dagegen kommt in diesem Roman Latein in Form von Sentenzen vor, um bestimmte Situationen zu illustrieren oder um Gedankengänge der Heldin auf indirekte Art zu verdeutlichen. So wird nicht explizit gesagt, dass sie sich gerne tragische Theaterstücke und Filme anschaut, sondern es wird mit einer Sentenz erklärt: „[...] sie hatte während des ganzen zweiten Akts von Ljubimovs Sonnenaufgängen geweint [...] das hielt sie nicht davon ab, später mit doppeltem Genuss an die Sonnenaufgänge ${ }^{10}$ zu denken, das ist nichts Seltsames, denn est quaedam flere voluptas. ${ }^{11 \text { “ }}(\mathrm{H}, 27)$. An einer anderen Stelle weist die lateinische Sentenz darauf hin, dass Helena, obwohl sie sich in estnischer Kultur schon gut orientiert, doch an ihren Wurzeln festhält: „[...] Liebling, du bist schon einigermaßen wie eine Estin geworden, sagte ihr einmal eine von ihren wenigen Bekannten in Tallinn, natürlich eine Russin; caelum, non animum mutant, qui trans mare currunt, ${ }^{12}$ hatte Helena entgegnet" $(H$, 113). Lateinische Sentenzen erscheinen also meistens an Stellen, wo Helenas Gedanken oder Stellungnahmen weitergegeben werden - möglicherweise, um ihre gewisse Wesenszüge, ihre Bildung und ihre Intelligenz zu illustrieren und ihre Ideen zu bekräftigen.

Laut Dembeck und Parr kann sich in einem Text neben der expliziten auch die implizite Mehrsprachigkeit manifestieren, zum Beispiel durch Übersetzung: „[...] wenn gesagt wird, eine Person spreche jetzt Spanisch, die Worte, in denen man diese Rede vor sich sieht, aber klar dem Englischen zugehören“" (Dembeck/Parr 2017: 10). Auch bei Markosjan-Käsper finden sich ähnliche

10 Es wird auf das Theaterstück Sonnenaufgänge angespielt, das von Juri Ljubimov $1971 \mathrm{im}$ Moskauer Taganka-Theater auf die Bühne gebracht wurde und das einen Meilenstein der russischen Theatergeschichte markiert.

11 „Auch das Weinen bietet eine gewisse Lust.“ Zitat aus Ovids Tristia, IV, 3, 37.

12 „Sie ändern den Himmel, nicht ihre Seele, die über das Meer eilen“. Zitat aus Horaz” Episteln, I, 11, 27. 
Beispiele der latenten Mehrsprachigkeit, auch wenn sie eher andeutungsweise zur Geltung kommen. In Penelope, die Listenreiche wird etwa erklärt, dass die Heldin und ihre Schwester miteinander Russisch sprechen, obwohl ihre Eltern Armenisch als Umgangssprache pflegen (P, 218). Es ist nicht ganz klar, in welcher Sprache Penelope mit ihren Eltern kommuniziert, doch vermutlich ist es Armenisch. Jedoch stehen im Text auch die Dialoge zwischen Penelope und ihren Eltern in der Originalsprache (Russisch). Aus Helena erfährt der Leser, dass die auf Armenisch erzogen wurde, jedoch in der Schule sehr gut Russisch lernte und deshalb diese Sprache als „eigene“ betrachtet $(\mathrm{H}, 106)$. Auch wird erklärt, dass Armenier gerne Russisch als Zweitsprache lernten und sie auch gut sprachen, im Gegensatz zu Esten, die zwar Russisch lernten, aber beim Sprechen „weder den hohen Stil noch die fließende Umgangssprache“ $(\mathrm{H}, 97)$ demonstrierten. ${ }^{13}$ Hierzu sollte erklärt werden, dass die sowjetische Sprachpolitik, die die Nationalsprachen unterdrückte, in Armenien teils mit Pragmatismus betrachtet wurde, denn der Abschluss einer russischen Schule und tiefe Kenntnis der russischen Sprache boten bessere Karrieremöglichkeiten (Grenoble 2003: 122f). Deshalb wurde für einen großen Teil der Schüler Russisch eine Bildungs- und Gelehrtensprache, während Armenisch für sie eine vorwiegend mündliche Umgangssprache blieb (Markosjan-Käsper 2006: 69). Die Folge jedoch war „das Sprachgemisch“, der eigentümliche „Mix, in dem sich halb Jerewan verständigte“ $(\mathrm{P}, 216)$ und die sprachlichen „Hybriden“ (P, 218).

Des Weiteren, so Dembeck und Parr, zeige sich in mehrsprachigen Texten auch „die Verwendung ,fremdsprachlicher' metrischer Muster, die ,wörtliche Übersetzung' anderssprachiger idiomatischer Wendungen, die Verwendung übersetzter anderssprachiger Zitate“, die eine andere Sprache innerhalb des Textes implizieren (Dembeck/Parr 2017: 11). Willms und Zemanek sehen solche Texte als Hybride, „gekennzeichnet durch Merkmale wie Heterogenität und Interferenz, Dialogizität und Polyphonie, Dezentralisierung und Subversion“ (Willms/Zemanek 2014: 3). Als Beispiel können hier die Sprachspiele, sprachliche Witze und Neuschöpfungen dienen, die in der deutschsprachigen transkulturellen Literatur von Yoko Tawada, Emine Sevgi Özdamar oder auch Aglaja Veteranyi oft eingesetzt werden (Wright 2008: 39-40; Willms/Zemanek 2014: 2). Auch Markosjan-Käsper benutzt Sprachspiele. In Penelope, die Listenreiche wird die Geschichte der Begegnung von

13 Es wird mit dem Unwillen der Esten erklärt, die Sprache der „Okkupanten“ zu lernen $(\mathrm{H}, 97)$, doch in der Wirklichkeit hängt es wohl eher mit sehr großen grammatischen und phonetischen Unterschieden zwischen estnischer und russischer Sprache und mit methodisch nicht gut durchdachten Lernmaterialien zusammen. 
Penelopes Eltern, Klara und Henrik, erzählt, und zwar anhand von russischen Zungenbrechern, die an dieser Stelle verballhornt werden:

Doch schon schwankten die Reihen der Neffen und Enkel, und die vier übergeschnappten Teufel traten ab, verkrümelten sich in ihre Zungenbrecher und krochen in den Zuber, der zurückgezogen am Zaun stand in dem Sommer, als Karl der Klara die Korallen klaute. So hatten sie sich auch kennen gelernt. (P, $44)^{14}$

Hierzu gehören auch Texte, in denen Systeme fremder Sprachen adaptiert werden oder eine Textsorte aus einer Sprache in eine andere übertragen wird, da in solchen Fällen die Herkunft aus einer anderen Sprache und damit aus einer anderen Kultur doch immer eingeschrieben bleibt, etwa bei Özdamar, die in ihren deutschen Texten türkische Syntax anwendet oder türkische Redewendungen übersetzt (Willms/Zemanek 2014: 2). Auch MarkosjanKäsper lässt ihre Figuren oft so sprechen, wie sie es in ihrer Muttersprache tun würden. So wird in Helena eine Tirade ihres Vaters Torgom weitergegeben, der darüber verärgert ist, dass seine Tochter sich die Leiden ihrer Patienten zu sehr zu Herzen nimmt: „[...] er nannte die Tochter einen kleinen Dummkopf: ,Du Dummkopf, oh, du Dummkopf, wem weinst du denn nach, mir und auch der Mutter ist nichts passiert, hör doch schon auf, bringst die Not ins Haus... “ (H, 28)

Neben der linguistischen Vielfalt in mehrsprachiger Literatur sollte meines Erachtens auch die Vielfalt von diversen kulturellen Praktiken erwähnt werden. Denn implizit werden in einem Text verschiedene kulturelle Traditionen eingebunden, die sich etwa im Textaufbau, in der Verwendung von bestimmten Hinweisen auf andere kulturell relevante Texte oder in bestimmten kulturellen Meta- oder Archecodes zeigen (Raud 2018: 130-141). Dies ist oft der Fall bei transkulturellen Autoren, deren Texte als eine Verflechtung von mehreren Sprach- und Kulturtraditionen und -schichten, die sich in mannigfachen Erscheinungen zeigen, angesehen werden können. Willms und Zemanek sprechen in diesem Zusammenhang auch von intertextueller bzw. textexterner Mehrsprachigkeit, der die Textgrenzen überschreitet (Willms/Zemanek 2014: 2).

14 In dem russischen Originalzitat sind die Zungenbrecher/Sprachspiele deutlicher zu erkennen: „Но уже Арогнули ряды племянников-внуков, и четыре чумазых черта отступими, упомзли в свою скороговорку посиживать на Аровах, так и не появившихся на траве, которой на Аворе не росло Ааже метом, когАа Карл у

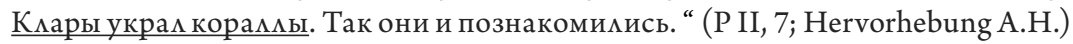


Verflochtene Sprachen und Kulturen

Dieses Phänomen kann anhand eines Zitats von Zafer Şenocak illustriert werden. Er beschreibt den Prozess, wie seine Zweisprachigkeit ihren Anfang nahm, wie folgt:

Das Mutterland Türkei hatte ich verlassen, doch das Spiegelland Türkei, [...], sollte mir erhalten bleiben. Diese Spiegelverkehrung war wichtig, ja unverzichtbar für die Entwicklung meiner literarischen Sprache und bildet letztendlich die Grundlage meiner Zweisprachigkeit. Ich schrieb auf Deutsch, aber mit türkischen Farbklängen im Ohr [...]. (Şenocak 2011: 78-79)

Das heißt, er schrieb auf Deutsch, doch manifestieren sich in seinen Texten türkische Kulturtraditionen auf eine natürliche und selbstverständliche Weise. Als ein weiteres Beispiel können die Erzählungen von Wladimir Kaminer gebracht werden. In seinen Büchern sehen wir die geschickte Verknüpfung von vielen Geschichten, die dem Erzähler oder seinen Bekannten angeblich passiert sind. In diesem Sinne pflegt Kaminer hier das Genre der so genannten бaйкa (bajka), der humoristischen Erzählung, die zwar die Wahrheit darzustellen beansprucht, gleichzeitig aber auch ziemlich frei mit konkreten Fakten und Daten operiert; aufgegriffen wird dabei die in der Sowjetunion weit verbreitete mündliche Tradition des Geschichten- und Anekdotenerzählens (vgl. Heero 2009: 222-223). Es werden also kulturelle Praktiken, die einer bestimmten Sprache eigen sind, erfolgreich in eine andere Sprache übertragen.

In Bezug auf Gohar Markosjan-Käsper sollte man noch einmal erwähnen, dass sie nicht als eine armenische oder russische Autorin klassifiziert werden wollte, sondern als eine Autorin, die bewusst europäische literarische Traditionen pflegt (Markosjan-Käsper 2006: 66-67). Deshalb scheint es logisch, dass die beiden hier behandelten Romane einen Bezugspunkt in der Weltliteratur haben. Penelopa ist wie die Spiegelverkehrung des Romans Ulysses von James Joyce (1922), in dem ein Tag im Leben (16.06.1904) der Hauptfigur Leopold Bloom beschrieben wird. Dargestellt werden nicht nur Ereignisse dieses Tages, sondern auch Gedanken, Vorstellungen, Assoziationen, die sowohl Bloom als auch die anderen Hauptfiguren dieses Romans erleben (Schwarz 1987: 2-4). Was den Roman Markosjan-Käspers betrifft, erfahren wir gleich zu Beginn, dass die Heldin Penelope einen Roman von Joyce, der eindeutig als Ulysses zu identifizieren ist, liest: „Penelope [...] zog [...] ein Bändchen Joyce zu sich heran. Von wegen Bändchen, einen fetten Band, noch dazu bis zum Geht-nichtmehr gefüllt mit in winzigster Schrift gedruckten Quatsch, der von Snobs in den Rang der intellektuellen Bibel erhoben wurde." (P, 8) So beginnt der Tag im Leben Penelopes, der im Weiteren detailliert beschrieben wird. Penelope, die hier quasi die Rolle des Odysseus übernimmt, irrt in diesem Roman nicht durch die Welt, sondern durch die ins postsozialistische Chaos gefallene 
Stadt Jerewan. Der Roman dokumentiert nicht nur ihre Aktivitäten im Laufe des Tages, sondern auch ihre Gedanken und Assoziationen, die in Form des inneren Monologs festgehalten werden, z.B.:

Ja. Ach. Am Morgen war alles in Ordnung. Und der Teufel hat dich geritten, Penelope, am anderen Ende anzufangen. Wärst du gleich hierhergekommen, wärst du jetzt gewaschen und appetitlich wie Ferkel Tschunja. Dabei hast du es nicht weit, zwei Häuserblocks! Bei Gott, das hat etwas Fatales. (P, 256)

Der Roman Helena dagegen nimmt Bezug auf Michail Bulgakovs Meister und Margarita (1928-1940). Die Parallelen im Aufbau dieser Romane sind sofort zu sehen. Bulgakovs Roman spielt parallel in Moskau in den 1930er Jahren und in Jerusalem um Ostern im Jahr 33 unserer Zeitrechnung; dargestellt wird einerseits ein satirisches Bild des Lebens in der Stalin-Ära und andererseits die „wahre“ Geschichte des Martyriums Jesu (bzw. Ha-Notsri). Der Roman Markosjan-Käspers spielt in Jerewan, Moskau und Tallinn gegen Ende der 1980er Jahre und parallel dazu wird die Geschichte einer Heldin der Antike, der Schönen Helena, erzählt, so wie sie möglicherweise wirklich war: Eine etwas vernachlässigte und gelangweilte Ehefrau findet einen Liebhaber (Paris) und zieht zu ihm. Dies setzt eine Kette von Ereignissen in Bewegung und hat den Trojanischen Krieg zur Folge. ${ }^{15}$ Auch wenn Markosjan-Käsper das Leben in Tallinn und Jerewan nicht so hart kritisiert, sind auch in ihrem Roman satirisch-ironische Momente durchaus sichtbar, bei denen oft direkt Bezug auf Bulgakovs Text genommen wird. Beispielsweise wird über die Esten gesagt: „Mit einem Wort, ganz gewöhnliche Menschen, und wie alle auch ein bisschen durch die Wohnungsfrage verdorben." $(\mathrm{H}, 73)^{16}$.

\section{Zum Schluss}

Die vorliegende Studie hat gezeigt, dass der „Kopf eines zweisprachigen Menschen [...] immer eine Raumerweiterung erfahren“ hat (Şenocak 2011: 20). Diese Feststellung konvergiert mit der neuesten Diskussion in der literarischen Öffentlichkeit in Armenien sowie in Estland. Laut Markosjan-Käsper gebe es

15 Wenn man Helena als Beispiel transkulturellen Schrifttums betrachtet, dann kann man auch hinzufügen, dass die Parallelgeschichte der Schönen Helena auch die Geschichte einer Frau ist, die wegen der Liebe ihr Leben in der Fremde verbringen muss und die sich in einer neuen Umgebung und Kultur zurechtfinden muss.

16 Mit diesen Worten charakterisiert Woland in Meister und Margarita die Einwohner Moskaus: sie seien gewöhnliche Leute, etwa solche Leute wie früher, nur durch die Wohnungsfrage verdorben (vgl. Bulgakov 1995: 138-139). 
ziemlich viele neue Autoren in der armenischen Diaspora, die in ihren Werken eher internationale Trends pflegen und die außer auf Russisch auch auf Englisch, Französisch oder Spanisch schreiben. Trotzdem werden sie als armenische Autoren betrachtet (Markosjan-Käsper 2006: 70). Auch in Estland sieht man besonders die jüngeren russischsprachigen Schriftsteller als eher international orientierte Autoren an, die die russischen Literaturtraditionen nicht mehr als Vorbild nehmen (Kotjuh 2012: 138-139; Kotjuh 2013: 69-77). Daher ist es lohnend, Gohar Markosjan-Käspers Werke sowie die Texte anderer estnischen russischsprachigen Autoren aus transkulturell und multilingual besetzter Perspektive zu untersuchen.

\author{
Aigi Heero \\ aheero@tlu.ee \\ Tallinn University \\ ESTONIA
}

\title{
Literatur
}

Allolio-Näcke, L., Kalscheuer, B., Manzeschke, A. 2005. Differenzen anders denken. Bausteine zu einer Kulturtheorie der Transdifferenz. Frankfurt/New York: Campus. Amodeo, I. 1996. Die Heimat heißt Babylon: Zur Literatur ausländischer Autoren in der Bundesrepublik Deutschland. Opladen: Westdeutscher Verlag.

Baumgärtel, B. 1997. Identitätsbalance in der Fremde: Der Beitrag des symbolischen Interaktionismus zu einem theoretischen Rahmen für das Problem der Identität in der Migrantenliteratur. - S. Fischer, M. McGowan, Hrsg., Denn du tanzt auf einem Seil. Positionen deutschsprachiger MigrantInnenliteratur. Tübingen: Stauffenburg Verlag, 53-69.

Belobrovtseva, I. 2018. Vene kirjandus Eestis: eile ja täna. - Looming, 1, 102-121.

Bulgakov, M. 1995 = Букгаков, М. 1995. Mactep и Mаргарита. Собачье сердие. Москва: Панорама.

Darieva, T. 2007. Migrationsforschung in der Ethnologie. - B. Schmidt-Lauber, Hrsg., Ethnizität und Migration. Einführung in Wissenschaft und Arbeitsfelder. Berlin: Reimer, 69-94.

Dembeck, T., Parr, R. 2017. Mehrsprachige Literatur: Zur Einleitung. - T. Dembeck, R. Parr, Hrsg., Literatur und Mehrsprachigkeit: Ein Handbuch. Tübingen: Narr Francke Attempto, 9-14.

Ernst, T. 2019. Von Mischkultur und Mehrsprachigkeit: Deutsch in der Literatur Luxemburgs. - Oxford German Studies, 48 (1), 91-112. https://doi.org/10.1080/ 00787191.2019 .1583444

Glesener, J. E. 2016. Migration Literature as a New World Literature? An Overview of the Main Arguments. -http://cielam.univ-amu.fr/node/1983 (27.01.2020). 
HEERO

Grenoble, L. A. 2003. Language Policy in the Soviet Union. New York etc: Kluwer Academic Publishers.

Heero, A. 2009. Zwischen Ost und West: Orte in der deutschsprachigen transkulturellen Literatur. - H. Schmitz, Hrsg., Von der nationalen zur internationalen Literatur. Transkulturelle deutschsprachige Literatur und Kultur im Zeitalter globaler Migration. Amsterdam: Rodopi, 205-225. https://doi.org/10.1163/9789042028777_013

Heero, A. 2019. On Cultural Contacts and their Impact on Literature in Tallinn in the Early 17th Century. - Philologia Estonica Tallinensis, 4, 143-160. https://doi. org/10.22601/PET.2019.04.07

Horaz 2011 = Quintus Horatius Flaccus 2011. Epistulae/Briefe. Stuttgart: Reclam.

Jürgenson, A. 2016. Assimilatsionistidest transnatsionalistideni - rände ja integratsiooni uurimine 20. ja 21. sajandil. - Tuna, 2, 91-105.

Kartau, M. 2015. Lahkus prosaist Gohar Markosjan-Käsper. - https://kultuur.err. ee/308199/lahkus-prosaist-gohar-markosjan-kasper (16.02.2020).

Katus, K., Puur, A., Põldma, A. 2002. Eesti põlvkondlik rahvastikuareng $=$ Cohort Population Development in Estonia. EKDK WP D-2. Tallinn: Eesti Kõrgkoolidevaheline Demouuringute Keskus/Estonian Interuniversity Population Research Centre.

Kotjuh, I. 2012. Eesti venekeelne kirjandus: kas osa eesti või vene kirjandusest? - Keel ja Kirjandus, 2, 134-139.

Kotjuh, I. 2013. Eesti venekeelse kirjanduse nullindate põlvkond: vastuvõtt ja tõrked omaks tunnistamisel. - Methis. Studia humaniora Estonica, 11, 64-83. https://doi. org/10.7592/methis.v8i11.1002

Lember, U. 2017. Unistus teistsugusest elust Nõukogude Läänes. - Müürileht 15.05.2017. https://www.muurileht.ee/unistus-teistsugusest-elust-noukogudelaanes/ (9.02.2020).

Lukas, L. 2008. Balti kirjakultuuri mitmekeelsest loomusest. - R. Undusk, Hrsg., Rahvuskultuur ja tema teised. Tallinn: Underi ja Tuglase Kirjanduskeskus, 23-33.

Markosjan-Kasper [sic], G. 2002. Penelope, die Listenreiche. Übersetz von Gabriele Leupold. Berlin: Rowohlt. [P]

Markosjan-Kasper [sic], G. 2020 = Маркосян-Каспер, Г. 2020. Пенелопа. Онлайн книга. - http://loveread.ec/read_book.php?id=39679\&p=1 (23.04.2020) [P II].

Markosjan-Käsper, G. 2004. Helena. Übersetz von Kalle Käsper. Faatum. [H]

Markosjan-Käsper, G. 2006. Teekond säravatele tippudele. Loomingu Raamatukogu, 26. Übersetz von Kalle Käsper. SA Kultuurileht.

Martinez Guillem, S. 2015. Migration Discourse. - K. Tracy, Hrsg., The International Encyclopedia of Language and Social Interaction. Hoboken: JohnWiley \& Sons. https://doi.org/10.1002/9781118611463.wbielsi100

Ovid = P. Ovidius Naso 2011. Briefe aus der Verbannung / Tristia. Epistulae ex Ponto. Berlin: De Gruyter.

Rabanus, S., Barseghyan, H. 2015. Language Choice and Identity in Post-Soviet Armenia. - P. Rosenberg, K. Jungbluth, D. Zinkhan Rhobodes, Hrsg., Linguistic Construction of Ethnic Borders. Frankfurt a. M. etc.: Peter Lang, 13-32. 
Verflochtene Sprachen und Kulturen

Raud, R. 2018. Tähenduste keeris. Tervikliku kultuuriteooria visand. Tallinn: TLÜ Kirjastus.

Sakkeus, L. 1994. The Baltic States. - S. Ardittis, Hrsg., The Politics of East-West Migration. New York: St. Martin’s Press, 68-85. https://doi.org/10.1007/978-1349-23352-6_4

Schwarz, D. R. 1987. Reading Joyce's Ulysses. New York: St. Martin's Press. https://doi. org/10.1007/978-1-349-18745-4

Şenocak, Z. 2011. Deutschsein. Eine Aufklärungsschrift. Hamburg: Edition KörberStiftung.

Spengler-Axiopoulos, B. 2002. Penelopes Tag. Ein armenischer Roman von Gohar Markosjan-Kasper. - Neue Zürcher Zeitung 16.05.2002. https://zeitungsarchiv. nzz.ch/neue-zuercher-zeitung-vom-16-05-2002-seite-67.html?hint $=11478695$ (16.02.2020).

Willms, W., Zemanek E. 2014. Polyglotte Texte. Formen und Funktionen literarischer Mehrsprachigkeit von der Antike bis zur Gegenwart. - Komparatistik Online, 2. https://www.komparatistik-online.de/index.php/komparatistik_online/issue/ view/9 (23.04.20).

Wright, C. 2008. Writing in the 'Grey Zone': Exophonic Literature in Contemporary Germany. - gfl-journal, 3, 26-42.

Zirnask, M., Püve, B. 2005. Elu ühes päevas. Gohar Markosjan-Käsper - Eesti Ekspress 2.06.2005. https://ekspress.delfi.ee/kuum/elu-uhes-paevas-gohar-markosjan-kasper?id=69068393 (16.02.2020). 